\title{
A Model to Improve Hospital-Based Palliative Care: The Palliative Care Redistribution Integrated System Model (PRISM)
}

\author{
Nauzley C. Abedini, MD ${ }^{1,2 *}$, Vineet Chopra, MD, MSc
}

${ }^{1}$ National Clinician Scholars Program, University of Michigan, Ann Arbor, Michigan; ${ }^{2}$ Division of Hospital Medicine, University of Michigan, Ann Arbor, Michigan.

Many hospitalized patients have unmet palliative care needs that are exacerbated by gaps in the palliative care subspecialty workforce. Training frontline physicians, including hospitalists, to provide primary palliative care has been proposed as one solution to this problem. However, improving palliative care access requires more than development of the physician workforce. System-level change and interdisciplinary approaches are also needed. Using task shifting as a guiding principle, we propose a new workforce framework (the Palliative care Redistribution Integrated System Model, or PRISM), which utilizes physician and nonphysician providers and resources to their maximum potential. We highlight the central role of hospitalists in this model and provide examples of innovations in screening, workflow, quality, and benchmarking to enable hospitalists to be purveyors of quality palliative care. Journal of Hospital Medicine 2018;13:868-871. Published online first August 29, 2018. (c) 2018 Society of Hospital Medicine

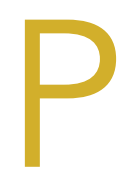

alliative care is an essential component of inpatient medicine. At its core, it is an interdisciplinary philosophy of care aiming to achieve the best quality of life for patients and families in the physical, psychosocial, and spiritual domains. With the aging population and growing complexity of hospitalized patients, inpatient palliative care needs are only projected to rise. However, a mismatch exists between the number of palliative care-trained physicians and the demand for such physicians. Currently, only 6,600 US physicians are board certified in palliative care-just $37 \%$ of the projected need. ${ }^{1}$ These workforce shortages have serious implications. In fact, it is estimated that nearly $40 \%$ of all hospitalized patients who need palliative care go without it. ${ }^{2}$

Existing efforts to improve access to palliative care have largely focused on bolstering the palliative care workforce. One tactic particularly relevant to hospitalists centers on frontline physicians providing "primary" palliative care: basic symptom management, patient-centered communication, and goals of care assessment, regardless of the disease state. ${ }^{3}$ Such physicians constitute the base of today's palliative care workforce model-a three-tiered pyramid built on clinician availability and skills. In this model, the second tier ("secondary" palliative care) includes physicians supported by a palliative care consultant or referral. The third level ("tertiary" palliative care) encompasses care provided directly by specialized palliative care teams, usually within academic medical centers (Figure 1). ${ }^{4}$

The practice of primary palliative care is central to the prac-

\begin{abstract}
Address for correspondence: Nauzley Abedini, MD, University of Michigan, Division of Hospital Medicine, UH South Unit 4, 1500 East Medical Center Drive, Ann Arbor, MI 48109-5220; Telephone: 425-922-4804; Email: nauzley@umich.edu
\end{abstract}

Received: March 8, 2018; Revised: April 27, 2018; Accepted: May 12, 2018

(๑) 2018 Society of Hospital Medicine DOI 10.12788/jhm.3065 tice of hospital medicine. ${ }^{5,6} \mathrm{After}$ all, hospitalists generate nearly half of all inpatient palliative care consultations ${ }^{7}$ and routinely interface with social workers, pharmacists, nurses, chaplains, and other consultants in their daily activities. Consequently, they are also well versed in serious illness communication and prognostication. ${ }^{8}$ In many ways, they are ideal purveyors of palliative care in the hospital.

Why then does the challenge to meet the demands of patients with palliative care needs persist? The truth may lie in at least three central shortcomings within the tiered palliative care workforce model. First, physicians comprising the base (where hospitalists typically fall) possess variable skills and knowledge in caring for seriously ill patients. While training opportunities exist for interested individuals, ${ }^{7}$ education alone can rarely achieve a systematic change. Second, some physicians may have the requisite skills but lack the time or resources to address palliative care needs. ${ }^{8}$ This is particularly true for inpatient clinicians who face pressures related to throughput and relative value units (RVUs). Third, the tiered approach is highly physician-centric, ignoring nonphysicians such as nurses, chaplains, and social workers outside of traditional palliative care subspecialty teams - members who are integral to the holistic approach that defines palliative medicine.

\section{THE PALLIATIVE CARE REDISTRIBUTION INTEGRATED SERVICE MODEL (PRISM)}

To better address the current palliative care access problem, we propose a new model: "The Palliative care Redistribution Integrated Service Model (PRISM; Figure 1)." Using the industrial engineering principle of "task shifting," this approach leverages disciplinary diversity and shifts specific activities from more specialized to less specialized members. ${ }^{9}$ In this way, PRISM integrates hospital-based interdisciplinary teams across all tiers of palliative care delivery. 

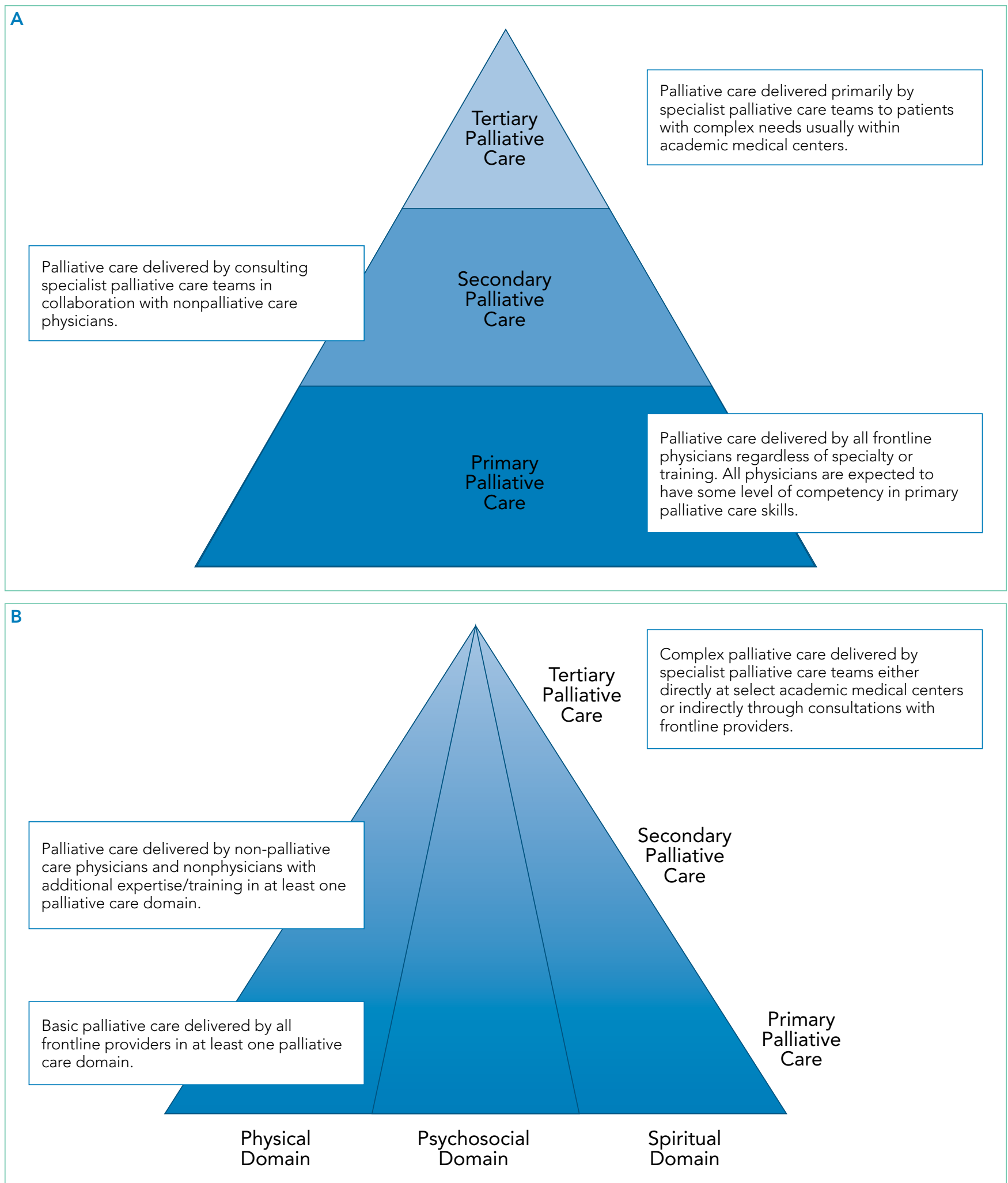

FIG 1. (A) The current 3-tiered pyramid model of palliative care workforce. (B) Palliative Care Redistribution Integrated System Model (PRISM): a new palliative care workforce model emphasizing task-shifting within three domains of palliative care-physical, psychosocial, and spiritual care.

PRISM sheds a tier-based approach in favor of flexible, skillbased verticals that span all physician and nonphysician providers. By dividing the original pyramid into three domains- physical, psychosocial, and spiritual-providers with various spheres of expertise may serve patients on multiple tiers. For example, a bedside nurse may perform basic psychosocial as- 
sessment consistent with his or her training, while physicians may focus on code status or prescribe antiemetics or low-dose opiate monotherapy-skills they have refined during medical school. Analogously, secondary palliative care may be delivered by any provider with more advanced skills in communication or symptom management. In this way, we expand the pool of clinicians available to provide palliative care to include nurses, hospitalists, oncologists, intensivists, social workers, and chaplains and also recognize the diversity of skill sets within and between disciplines. Thus, a hospitalist may clarify the goals of care but may ask a social worker trained in psychosocial assessment for assistance with difficult family dynamics or a chaplain for spiritual needs. Interdisciplinary teamwork and cross-disciplinary communication-hallmarks of palliative care-are encouraged and valued. Furthermore, if providers feel uncomfortable providing a certain type of care, they can ask for assistance from more experienced providers within their discipline or outside of it. In rare cases, the most complex patients may be referred to specialist palliative care teams.

Inherent within PRISM is a recognition that all providers must have a basic palliative care skillset obtained through educational initiatives. ${ }^{7}$ Yet focusing solely on training the workforce as a strategy has and will continue to miss the mark. Rather, structural changes to the means of providing care are also needed. Within hospitals, these changes often rely heavily on hospitalists due to their central position in care delivery. In this way, hospitalists are well primed to be the agents of change in this model.

\section{The Role of Technology}

Since many hospitalized patients have unrecognized and underserved palliative care needs, a formal approach to assessment is needed. Lin et al. proposed criteria for a "sentinel hospitalization," marking a major illness or transition in highrisk patients necessitating palliative interventions. ${ }^{10}$ Similar screening criteria have been validated among hospitalized oncology patients ${ }^{11}$ and in critical care. ${ }^{12}$ While checklists have been shown to help identify hospitalized patients with palliative care needs, ${ }^{13}$ their implementation has been slow, presumably because they are burdensome for busy providers to complete.

Technological automation may be a solution to the checklist conundrum. For example, if palliative care screening criteria could be automatically extracted from electronic health records, scoring systems could trigger hospitalists to consider the goals of care discussions or engage an interdisciplinary care team to fulfill a variety of needs. Frameworks for such scoring systems already exist and are familiar to most hospitalists. For example, admission order sets routinely calculate the Padua or Caprini score to facilitate decision-making for prophylaxis of deep vein thrombosis. An admission order set that screens and prompts decision-making around palliative care needs is thus feasible. One example is a hard stop for entering code status in the admission order set; in turn, this hard stop could also trigger providers to complete a "check-box" palliative care screening checklist. Automatic extraction of certain data from the record-such as age, prior code status, recent hospitalizations, or mobility scores-could auto-populate to facilitate decision-making. In turn, measuring the influence of such tools on access to palliative care, workflow, and capacity will be important, as most tools may not have quality or value intended. ${ }^{14}$

\section{Streamlining Workflow}

It is common for hospitalists to oversee care for 15-20 patients at a time. Thus, they may not have the time to meaningfully engage patients to assess palliative care needs. Creating designated hospitalist palliative care teams with enhanced interdisciplinary support for patients identified using sentinel hospitalization or checklist-based tools may help to solve this dilemma. These teams may also employ lower "caps," freeing up time for critical discussions and planning around end of life. At the University of Michigan, we are planning just such an approach, a strategy which has the additional benefit of bypassing the binary "care versus no care" dilemma faced by patients choosing palliation. Rather, patients may continue to receive treatments congruent with the goals of care in such teams.

\section{Making Palliative Care a Standard of Care}

A call for health systems to develop and implement palliative care quality metrics has emerged. Given their role in quality improvement and health system reform, hospitalists are well positioned to shepherd this imperative. Creating incentives to screen inpatients for palliative care needs and develop new homes in which to care for these patients are but a few ways to help set the tone. Additionally, developing and sharing quality metrics and benchmarks currently captured in repositories such as the Palliative Care Quality Network, Global Palliative Care Quality Alliance, and Center to Advance Palliative Care can help to assess and continually improve care delivery. Creating and sharing dashboards from these metrics with all providers, regardless of discipline or training, will ensure accountability to deliver quality palliative care.

\section{CONCLUSION}

Many hospitalized patients do not receive appropriate attention to their palliative care needs. A new interdisciplinary workforce model that task shifts to physician and nonphysician providers and pairs system-level innovations and quality may solve this problem. Input and endorsement from a wide variety of disciplines (particularly our nonphysician colleagues) are needed to make PRISM operational. The proof of concept will lie in testing feasibility among key stakeholders and rigorously studying the proposed interventions. Through innovation in technology, workflow, and quality improvement, hospitalists are well poised to lead this change. After all, our patients deserve nothing less.

Disclosures: The authors have nothing to disclose.

Funding: Dr. Abedini's work is supported by the University of Michigan National Clinician Scholars Program at the Institute for Healthcare Policy and Innovation, as well as the University of Michigan Division of Hospital Medicine. 


\section{References}

1. Lupu D. American Academy of Hospice and Palliative Medicine Task Force Estimate of current hospice and palliative medicine physician workforce shortage. J Pain Symptom Manage. 2010;40(6):899-911. doi: 10.1016/j.jpainsymman.2010.07.004

2. Chuang E, Hope AA, Allyn K, Szalkiewicz E, Gary B, Gong MN. Gaps in provision of primary and specialty palliative care in the acute care setting by race and ethnicity. J Pain Symptom Manage. 2017;54(5):645-653. doi: 10.1016/j. jpainsymman.2017.05.001

3. Quill TE, Abernethy AP. Generalist plus specialist palliative care--creating a more sustainable model. N Engl J Med. 2013;368(13):1173-1175. doi: 10.1056/NEJMp1215620

4. von Gunten CF. Secondary and tertiary palliative care in US hospitals. JAMA 2002;287(7):875-881. doi: 10.1001/jama.287.7.875

5. Pantilat SZ. Hope to reality: the future of hospitalists and palliative care. $J$ Hosp Med. 2015;10(10):701-702. doi: 10.1002/jhm.2401

6. Meier DE. Palliative care in hospitals. J Hosp Med. 2006;1(1):21-28. doi: 10.1016/j.cger.2004.07.006

7. Fail RE, Meier DE. Improving quality of care for seriously ill patients: Opportunities for hospitalists. J Hosp Med. 2018;13(3):194-197. doi: 10.12788/ jhm.2896. [Epub ahead of print]

8. Rosenberg LB, Greenwald J, Caponi B, et al. Confidence with and barriers to serious illness communication: A national survey of hospitalists. J Palliat Med. 2017;20(9):1013-1019. doi: 10.1089/jpm.2016.0515

9. Carayon P, Gurses AP. Nursing workload and patient safety-a human factors engineering perspective. In: Hughes RG, ed.Patient Safety and Quality: An Evidence-Based Handbook for Nurses. Rockville, MD: Agency for Healthcare Research and Quality (US); 2008.

10. Lin RJ, Adelman RD, Diamond RR, Evans AT. The sentinel hospitalization and the role of palliative care. J Hosp Med. 2014;9(5):320-323. doi: 10.1002/jhm.2160

11. Glare PA, Chow K. Validation of a simple screening tool for identifying unmet palliative care needs in patients with cancer. J Oncol Pract. 2015;11(1):e81-e86. doi: 10.1200/JOP.2014.001487.

12. Zalenski RJ, Jones SS, Courage $C$, et al. Impact of a palliative care screening and consultation in the ICU: A multihospital quality improvement project. J Pain Symptom Manage. 2017;53(1):5-12.e3. doi: 10.1016/j.jpainsymman.2016.08.003.

13. Weissman DE, Meier DE. Identifying patients in need of palliative care assessment in the hospital setting: a consensus report from the Center to Advance Palliative Care. J Palliat Med. 2011;14(1):17-23. doi:

14. MacLean $\mathrm{CH}$, Kerr EA, Qaseem A. Time out-charting a path for improving performance measurement. N Engl J Med. 2018. Epub ahead of print. doi: 10.1056/NEJMp1802595 